This is a self-archived version of an original article. This version may differ from the original in pagination and typographic details.

Author(s): Kukkonen, Esa Petteri; Lahtinen, Elmeri; Myllyperkiö, Pasi; Konu, Jari; Haukka,

Title: Three-Dimensional Printing of Nonlinear Optical Lenses

Year: 2018

Version: Published version

Copyright: @ 2018 American Chemical Society

Rights: In Copyright

Rights url: http://rightsstatements.org/page/lnC/1.0/?language=en

Please cite the original version:

Kukkonen, E. P., Lahtinen, E., Myllyperkiö, P., Konu, J., \& Haukka, M. (2018). Three-Dimensional Printing of Nonlinear Optical Lenses. ACS Omega, 3(9), 11558-11561.

https://doi.org/10.1021/acsomega.8b01659 


\title{
Three-Dimensional Printing of Nonlinear Optical Lenses
}

\author{
Esa Kukkonen, ${ }^{\dagger}$ Elmeri Lahtinen, ${ }^{\dagger}$ Pasi Myllyperkiö, ${ }^{\dagger \odot}$ Jari Konu, ${ }^{\dagger}{ }^{\dagger}$ and Matti Haukka ${ }^{*}{ }^{\dagger}$ \\ ${ }^{\dagger}$ Department of Chemistry and ${ }^{\ddagger}$ Nanoscience Center, University of Jyväskylä, P.O. Box 35, FI-40014 Jyväskylä, Finland
}

\section{Supporting Information}

\begin{abstract}
In the current paper, a series of nonlinear optical (NLO) active devices was prepared by utilizing stereolithographic three-dimensional printing technique. Microcrystalline NLO active component, urea, or potassium dihydrogen phosphate was dispersed in a simple photopolymerizable polyacrylate-based resin and used as the printing material to fabricate highly efficient transparent

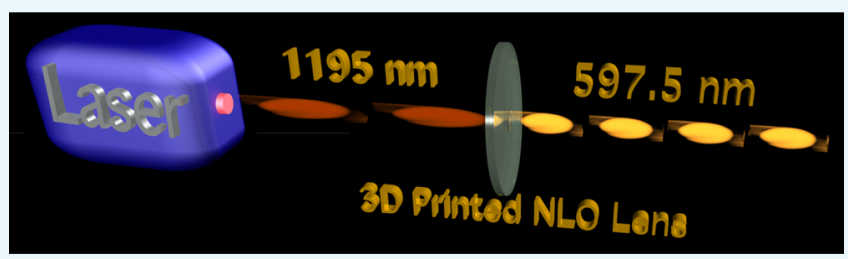
NLO lenses. The nonlinear activity of the printed lenses was confirmed by second-harmonic generation measurements using a femtosecond laser-pumped optical parametric amplifier operating at a wavelength of $1195 \mathrm{~nm}$. The three-dimensional printing provides a simple method to utilize a range of NLO active compounds without tedious crystal growing and processing steps. Furthermore, introducing NLO additives in the printing material provides an easy and cost-efficient way to manufacture lenses with NLO functionality.
\end{abstract}

\section{INTRODUCTION}

The research on nonlinear optical (NLO) materials dates back to the early days of lasers. Franken et al. reported the observation of second-harmonic generation (SHG) phenomenon already in $1961 .^{1}$ In SHG, two photons having the same frequency are combined in a medium generating radiation that has twice the frequency ("frequency doubling") and thus half the wavelength of the original photons. Half a century later, SHG still remains as one of the most investigated NLO property owing to its potential applications in laser technology and optoelectronics. ${ }^{2,3}$ Especially, the growing interest in processes such as optical signal processing and electro-optical switches ${ }^{4}$ has been fueling the research in the field of NLO materials and devices. ${ }^{5,6}$

Most commonly used NLO devices contain large single crystals of the active material. Although a variety of NLO active materials, both inorganic and organic, are known, only a limited number of these are commercially used. The advantages of the most commonly used inorganic compounds, such as $\beta$-barium borate ${ }^{7}$ and potassium titanyl phosphate, ${ }^{8,9}$ arise from the wide-transparency regions, wide phase matching range, and high conversion efficiency, as well as high physical and chemical stability and durability. Due to these reasons, the selection of commercial materials has remained nearly unchanged for decades even though numerous new inorganic and organic materials have shown superior NLO properties. $^{10-12}$ Especially, various organic compounds have displayed a number of NLO properties that clearly exceed that of the commercial inorganic crystals. ${ }^{10-14}$ Unfortunately, many of these organic NLO components suffer from inferior stability and malleability, which has hindered their utilization in optical devices. However, the stability problems of certain NLO films have been successfully tackled by using specific preparation techniques based on self-assembly of the NLO compounds. ${ }^{15,16}$ Despite the steps taken forward, assembling molecules or the need to grow single crystals of high quality and substantial size have still limited the use of wider selection of organic compounds. Crystallization techniques such as topseeded solution growth technique, Czochralski technique, and hydrothermal methods, commonly used for inorganic compounds, are often less suitable for organic materials. ${ }^{17,18}$ Furthermore, the mechanical processing of NLO crystals, required to meet the demands of optical components, may be inapplicable for organic crystals. One way around these problems is to fabricate thin films by the poling technique where the organic NLO chromophore is mixed with a polymer matrix. In this technique, the dipoles of the NLO components are aligned by an external electric field. ${ }^{19,20}$ Again, this method is not suitable for all organic NLO compounds. Another potential solution would be utilization of microcrystalline materials, instead of large single crystals, as in the Kurtz-Perry technique, which is used for measuring SHG properties. ${ }^{21}$ However, the powdery material is difficult to handle and therefore poorly applicable for practical NLO use as it is.

In this paper, we present a method for manufacturing NLO active devices from microcrystalline NLO components by three-dimensional (3D) printing. In this method, the chromophore microcrystals, such as urea or potassium dihydrogen phosphate (KDP), are mixed with photopolymerizable polyacrylate-based resin, commonly used as printing material in stereolithographic (SLA) 3D printing technique. The 3D printing opens possibilities of tailor-made size, shape and, optical properties of the printed objects. The 3D printing has already been successfully used for printing high-quality lenses. $^{22}$ By using an NLO additive in the printing material, it is possible to prepare lenses with NLO properties. Use of

Received: July 14, 2018

Accepted: September 10, 2018

Published: September 21, 2018 


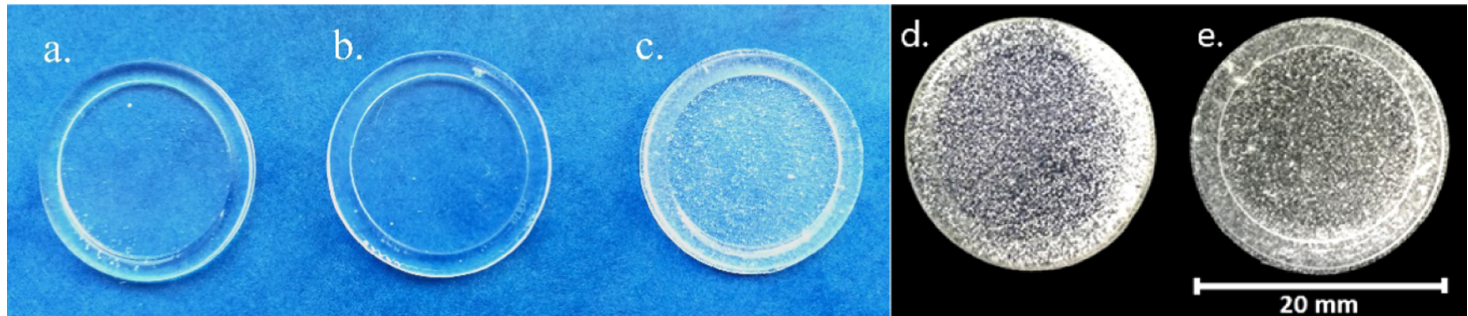

Figure 1. Photographs (blue background) and optical microscope images (black background) of the printed lenses. (a) The $300 \mu \mathrm{m}$ thick reference lens without the NLO component. (b, d) The $300 \mu \mathrm{m}$ thick NLO lens with 5 wt $\%$ KDP. (c, e) The $300 \mu \mathrm{m}$ thick NLO lens with 5 wt $\%$ urea.

polymer matrix also serves as a protecting media for the NLO component. This makes it possible to utilize a whole range of less stable organic compounds as NLO chromophores.

\section{RESULTS AND DISCUSSION}

The 3D printing experiments were conducted by adding microcrystalline urea or potassium dihydrogen phosphate (KDP) as the NLO active component in the printable photopolymer matrix. Both of these highly effective NLO chromophores are commonly used reference materials in the SHG measurements carried out by the Kurtz-Perry powder method. ${ }^{21,23,24}$ A series of round NLO lenses with $5 \mathrm{wt} \%$ urea or KDP were printed by SLA technique. The diameter of the printed objects was $2 \mathrm{~cm}$, and the thicknesses 300, 600, 900, 1200, and $1500 \mu \mathrm{m}$ (Figure 1). As can be seen in Figure 1, both additives were uniformly dispersed throughout the objects. Furthermore, the additives retained their original microcrystalline structure even after the printing process.

The 3D printed NLO lenses' ability to generate the secondharmonic signal was confirmed by measurements with an amplified femtosecond laser (Integra-C, Quantronix)-pumped tunable optical parametric amplifier (TOPAS, Light Conversion Ltd.) operating at $1195 \mathrm{~nm}$. A laser beam of $3 \mathrm{~mm}$ (full width at half maximum, FWHM), a 130 fs pulse width, and 8 $\mu \mathrm{J}$ of pulse energy was aimed at the middle of each object, and the intensity of the second-harmonic wavelength generated was measured through the accumulation of 10 consecutive pulses. Relatively wide laser beam was used to obtain an average result covering the whole lens.

With both urea and KDP lenses, the intensity of the SHG signal increased with the increasing thickness of the object (Figures S1, S2 and Table S1). The intensities of the SHG signals relative to the thickness of the lens are shown in Figure 2. With KDP, the intensity grew nearly linearly. The small

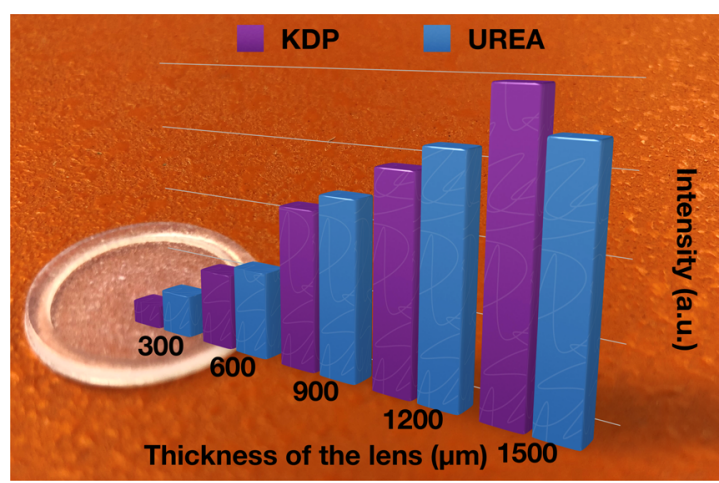

Figure 2. SHG intensities based on the integrated surface areas of the SHG signals for KDP and urea lenses as the function of lens thickness. deviations from the linear growth are likely due to inherently random orientations of the microcrystals inside the NLO lens. In the case of urea, the linear growth started to level after 1200 $\mu \mathrm{m}$ thickness. After certain thickness, the increased amount of microcrystals, crystal-to-crystal interactions, and decreasing transparency effectively reduce the intensity more than the new microcrystals increase it.

Organic polymers typically absorb UV radiation. Depending on the material, the absorption cutoff point can be reached already in the visible region. This must be kept in mind when choosing the matrix polymer. Therefore, the UV/vis spectrum of the printed acrylate polymer was measured in the range of 890-300 nm (Figures S3 and S4). Significant absorption started at ca. $420 \mathrm{~nm}$, indicating the apparent cutoff wavelength for this material. Although the absorption of the polymer sets the limit for the usable wavelengths, it does not necessarily set additional limitations in the case of objects with organic NLO chromophores. It should be noted that also the organic NLO components tend to absorb strongly in the UV region. ${ }^{25,26}$ Consequently, a similar cutoff would occur even without the polymer matrix.

The results show that stereolithography $3 \mathrm{D}$ printing can be utilized to produce highly efficient NLO lenses by using simple and readily available materials. Both urea- and KDP-based objects displayed significant ability to generate secondharmonic signal. The 3D printing method introduced here provides a way to considerably extend the selection of NLO active compounds that can be used for practical NLO devices. One of the main advantages of this novel approach is that there is no need for tedious preprocessing steps usually required when conventional NLO crystals are manufactured. Use of polymer matrix can also protect the highly efficient but less stable organic NLO compounds, which makes also those materials available for NLO applications. The ability to design the shape and size of the NLO lenses makes 3D printing a powerful tool for both research and manufacturing of new and improved electro-optical devices. It is already well established that 3D printing can be used to produce lenses with highquality optical properties. We believe that by combining the choice of efficient NLO compounds with the optical design of the object can open up a whole new era in manufacturing NLO lenses.

\section{METHODS}

Materials. The acrylate-based resin used in 3D printing was purchased from PeoPoly. Urea was purchased from SigmaAldrich (ACS Reagent, 99.0-100.5\%) and potassium dihydrogen phosphate (KDP) from Merck ( $\geq 99.5 \%)$. All chemicals were used as received. High-purity water of 18.2 $\mathrm{M} \Omega \mathrm{cm}$ resistivity was used throughout the experiments. 
Preparation of the NLO Lenses. Approximately, $55 \mathrm{~mL}$ of a commercial acrylate-based resin was weighed in a beaker and mixed with 5 wt \% urea or KDP. The NLO components had been sifted through $125 \mu \mathrm{m}$ sieve to obtain uniform crystal sizes. The mixtures were carefully stirred before printing until a homogeneous printing material was obtained. The mixture was used for SLA 3D printing immediately after the preparation. After the 3D printing, the objects were first washed thoroughly with ethanol and water and then cured using $405 \mathrm{~nm}$ UV lamp for $10 \mathrm{~min}$ to make sure that the polymerization was complete. The fully cured printed objects were coated with sprayed lacquer to improve the transparency.

Three-Dimensional Printing. The models of the NLO lenses were designed by FreeCad v.0.16 software. The PeoPoly version of the Cura 2.6.2.-14 and B9Creator v.1.1.0. was used for preparing the models for printing. All objects were printed with the PeoPoly Moai SLA 3D printer operating with a 150 $\mathrm{mW} 405 \mathrm{~nm}$ laser. The layer height used for preparation of the objects was $50 \mu \mathrm{m}$.

NLO Measurements. The SHG measurements were conducted using a widely tunable optical parametric amplifier (TOPAS, Light Conversion Ltd.) pumped by an amplified femtosecond laser (Integra-C, Quantronix). OPA signal beam output at $1195 \mathrm{~nm}$ was used to generate second harmonic on the samples. The beam size at the sample was $3 \mathrm{~mm}$ (FWHM). The pulse duration was $\sim 130 \mathrm{fs}$, and pulse energy was $8 \mu \mathrm{J}$. Exposure time of $0.217 \mathrm{~s}$ was used for urea lenses, whereas 2.17 $\mathrm{s}$ was used for KDP lenses.

UV/Vis Measurements. UV/vis spectrum of the printed polymer was measured from 890 to $300 \mathrm{~nm}$ using a PerkinElmer Lambda $25 \mathrm{UV} /$ vis spectrophotometer. The analyzed NLO lens used as a sample was placed in front of the sample cuvette holder, and the reference cuvette holder was left empty as air was used as reference. A slit of $1.0 \mathrm{~nm}$ was used, with a scan speed of $240 \mathrm{~nm} / \mathrm{min}$ and a data interval of $5.0 \mathrm{~nm}$.

\section{ASSOCIATED CONTENT}

\section{S Supporting Information}

The Supporting Information is available free of charge on the ACS Publications website at DOI: 10.1021/acsomega.8b01659.

Observed SHG intensities of the 3D printed lenses and $\mathrm{UV} /$ vis absorbance spectra of the lenses (PDF)

\section{AUTHOR INFORMATION}

\section{Corresponding Author}

*E-mail: matti.o.haukka@jyu.fi.

\section{ORCID}

Pasi Myllyperkiö: 0000-0003-1651-1676

Jari Konu: 0000-0002-7408-8995

Matti Haukka: 0000-0002-6744-7208

\section{Author Contributions}

M.H. and J.K. conceptualized the utilization of 3D printing for nonlinear optics and supervised the work. E.K. and E.L. printed and processed the objects. E.L. did the three-dimensional modeling. P.M. conducted the SHG measurements with E.K. and E.L. The paper was written jointly by E.K., E.L., J.K., and M.H.

\section{Notes}

The authors declare no competing financial interest.

\section{ACKNOWLEDGMENTS}

Financial support received from the Centennial Foundation of Technology Industries of Finland and Jane and Aatos Erkko foundation as a part of The Future Makers program is greatly appreciated.

\section{REFERENCES}

(1) Franken, P. A.; Hill, A.; Peters, C.; Weinreich, G. Generation of optical harmonics. Phys. Rev. Lett. 1961, 118.

(2) Wang, Y.; Pan, S. Recent development of metal borate halides: Crystal chemistry and application in second-order NLO materials. Coord. Chem. Rev. 2016, 323, 15-35.

(3) Cho, M. J.; Choi, D. H.; Sullivan, P. A.; Akelaitis, A. J. P.; Dalton, L. R. Recent progress in second-order nonlinear optical polymers and dendrimers. Prog. Polym. Sci. 2008, 33, 1013-1058.

(4) Garmire, E. Nonlinear optics in daily life. Opt. Express 2013, 21, No. 30532

(5) Mutailipu, M.; et al. Chemical Cosubstitution-Oriented Design of Rare-Earth Borates as Potential Ultraviolet Nonlinear Optical Materials. J. Am. Chem. Soc. 2017, 139, 18397-18405.

(6) Mutailipu, M.; et al. $\mathrm{SrB}_{5} \mathrm{O}_{7} \mathrm{~F}_{3}$ Functionalized with $\left[\mathrm{B}_{5} \mathrm{O}_{9} \mathrm{~F}_{3}\right]^{6-}$ Chromophores: Accelerating the Rational Design of Deep-Ultraviolet Nonlinear Optical Materials. Angew. Chem., Int. Ed. 2018, 57, 60956099.

(7) Chuantian, C.; Bochang, W.; Aidong, J.; Guiming, Y. A NewType Ultraviolet SHG Crystal $-\beta-\mathrm{BaB}_{2} \mathrm{O}_{4}$. Sci. Sin., Ser. B 1985, 28, 235-243.

(8) Zumsteg, F. C.; Bierlein, J. D.; Gier, T. E. $\mathrm{K}_{\mathrm{x}} \mathrm{Rb}_{1-\mathrm{x}} \mathrm{TiOPO}_{4}$ : A new nonlinear optical material. J. Appl. Phys. 1976, 47, 4980-4985.

(9) Bierlein, J. D.; Vanherzeele, H. Potassium titanyl phosphate: properties and new applications. J. Opt. Soc. Am. B 1989, 6, 622.

(10) Tam, W.; Guerin, B.; Calabrese, J. C.; Stevenson, S. H. 3Methyl-4-methoxy-4'-nitrostilbene (MMONS): Crystal structure of a highly efficient material for second-harmonic generation. Chem. Phys. Lett. 1989, 154, 93-96.

(11) Pan, F.; Wong, M. S.; Bosshard, C.; Günter, P. Crystal growth and characterization of the organic salt $4-\mathrm{N}, \mathrm{N}$-dimethylamino-4' $-\mathrm{N}$ methyl-stilbazolium tosylate (dast). Adv. Mater. 1996, 8, 592-595.

(12) Marder, S. R.; Perry, J. W.; Yakymyshyn, C. P. Organic Salts with Large Second-Order Optical Nonlinearities. Chem. Mater. 1994, $6,1137-1147$.

(13) Ledoux, I.; Lepers, C.; Périgaud, A.; Badan, J.; Zyss, J. Linear and nonlinear optical properties of N-4-nitrophenyl L-prolinol single crystals. Opt. Commun. 1990, 80, 149-154.

(14) Marder, S. R. Organic nonlinear optical materials: where we have been and where we are going. Chem. Commun. 2006, 131-134.

(15) Heflin, J. R.; et al. Efficient, Thermally Stable, Second Order Nonlinear Optical Response in Organic Hybrid Covalent/Ionic SelfAssembled Films. Langmuir 2006, 22, 5723-5727.

(16) Zhu, P.; et al. Realization of Expeditious Layer-by-Layer Siloxane-Based Self-assembly as an Efficient Route to Structurally Regular Acentric Superlattices with Large Electro-optic Responses. Chem. Mater. 2002, 14, 4982-4989.

(17) Dhanaraj, G.; Byrappa, K.; Prasad, V.; Dudley, M. Springer Handbook of Crystal Growth; Springer, 2010.

(18) Czochralski, J. Ein neues Verfahren zur Messung der Kristallisationsgeschwindigkeit der Metalle. Z. Phys. Chem. 1918, 92U, 219-221.

(19) Lee, H. S.; et al. Ellipsometric study of the poling effect on nonlinear-optical side-chain polymers containing disperse red 1 . J. Appl. Phys. 2007, 102, No. 013514.

(20) Gaidukovs, S.; Kampars, V.; Rutkis, M.; Nitis, E.; Tokmakovs, A. Poled nonlinear polymeric material, Patent EP2824509A1, 2013.

(21) Kurtz, S. K.; Perry, T. T. A Powder Technique for the Evaluation of Nonlinear Optical Materials. J. Appl. Phys. 1968, 39, 3798. 
(22) Gawedzinski, J.; Pawlowski, M. E.; Tkaczyk, T. S. Quantitative evaluation of performance of three-dimensional printed lenses. Opt. Eng. 2017, 56, No. 084110.

(23) Jerphagnon, J.; Kurtz, S. K. Optical Nonlinear Susceptibilities: Accurate Relative Values for Quartz, Ammonium Dihydrogen Phosphate, and Potassium Dihydrogen Phosphate. Phys. Rev. B 1970, 1, 1739-1744.

(24) Halbout, J.-M.; Blit, S.; Donaldson, W.; Chung, Tang Efficient phase-matched second-harmonic generation and sum-frequency mixing in urea. IEEE J. Quantum Electron. 1979, 15, 1176-1180.

(25) Uchida, T.; et al. Novel Organic SHG Materials. Mol. Cryst. Liq. Cryst. Sci. Technol., Sect. A 1998, 315, 135-140.

(26) Patil, P. S.; et al. Second harmonic generation and crystal growth of new chalcone derivatives. J. Cryst. Growth 2007, 303, 520524. 